\title{
Einleitung: Aras Ören - Zeitzeuge, Chronist und Archivar (West)Berlins
}

\author{
ELA GEZEN \\ University of Massachusetts Amherst
}

\begin{abstract}
Ich bin Türke, geboren 1939 in Istanbul. / Mein Paß trägt die Nummer TR-B 295136. / Bin 1,85 m groß, wiege 78 Kilo. / Mitten auf dem Kopf habe ich eine Glatze, meine Augenbrauen / aber sind buschig; / als besonderes Merkmal habe ich eine große Nase / und einen Schnauzbart (manchmal lasse ich mir auch einen / Vollbart stehen) / ich kam in diese Stadt aus freien Stücken, / ließ mich hier am 09. September 1969 nieder. / Die Gründe für mein Privatexil / möchte ich lieber für mich behalten. Ich glaube, was ich / erzähle genügt euch. (Ören, Dazwischen 38)
\end{abstract}

In diesen Versen gibt der Schriftsteller, Poet, Essayist, Theatermacher und Aktivist Aras Ören lyrisch seinen Lebenslauf wieder, mit sichtbarem Schwerpunkt auf sein Äußeres. Doch er erwähnt auch seine Einwanderung nach Westberlin, die direkt an sein Schreiben, das „Erzählen“, gebunden ist. Sein „Privatexil“, welches seine Auswanderung aus der Türkei wie auch seinen Aufenthalt in Deutschland umfasst, dient als Titel für zwei seiner Publikationen, der 1977 veröffentlichten Gedichtsammlung Privatexil und seiner 1999 erschienenen Tübinger Poetikvorlesungen Privatexil: Ein Programm? Drei Vorlesungen. In seiner zweiten Tübinger Vorlesung, ,Selbstbild mit Stadt“, geht er näher auf den von ihm geschaffenen Neologismus „Privatexil“ ein:

Mein privates Exil würde erst an dem Tag zu Ende gehen, an dem ich meine neue Heimat für mich erobern, sie als meine tatsächliche neue Heimat empfinden würde. Das würde hier, in Berlin stattfinden. Ich würde sie, meine neue Heimat schreibend entdecken. Dies war das Ziel und der Sinn meiner schriftstellerischen Tätigkeit. [...] Das konkrete Ergebnis [...]: Die Auseinandersetzung mit dem fremden Blick eines Exilanten auf die Stadt, als eine Reihe von geschriebenen Werken, deren Entdeckungsreichtum nicht zu unterschätzen ist. (39)

Die Zentralität (West)Berlins für sein literarisches Werk, Örens Wahrnehmung und auch seine Verbundenheit mit dieser Stadt, die er sich schreibend 
aneignet, sowie auch Zusammenhänge seiner Texte, werden hier erkennbar. Letzteres wird durch diese Passage insbesondere veranschaulicht, in dem sie das Verhältnis seiner Texte zueinander durch die prosaische Ergänzung der lyrischen Einführung des „Privatexils“ exemplifiziert. Darüber hinaus konstituiert er seine literarische Perspektive auf die Stadt als ,fremden Blick eines Exilanten“, jedoch ist fremd hier nicht als nicht-dazugehörig und außenstehend, sondern als bereichernd und somit positiv konnotiert, was gleichzeitig in der Aneignung der Stadt und einer literarischen Stadtgeschichte resultiert.

Aras Ören hat in verschiedenen Aufsätzen, Interviews und teilweise auch in seinem literarischen Oeuvre seine Poetik explizit formuliert - wie den vorangegangenen Zeilen entnommen werden kann. Im Rahmen dieser Einleitung möchte ich auf Teilaspekte seiner Poetikvorlesungen eingehen (mit einem Schwerpunkt auf der frühen Phase seiner literarischen Tätigkeit): In diesen hat er sich nicht nur zum Stellenwert und der Verortung türkischdeutscher Literatur innerhalb der deutschen Literaturlandschaft geäußert, sondern auch zur Signifikanz der türkischen Einwanderung für die Literatur allgemein und für Westberlin im Besonderen. Dies geschieht unter Berücksichtigung seiner eigenen Migrationserfahrungen und der Frage, inwiefern diese sein literarisches Oeuvre nicht nur nachhaltig beeinflusst, sondern auch bedingt haben. Es werden weitere Texte herangezogen, die in den Vorlesungen ausgeführte Ansätze und Zusammenhänge erkennen lassen und somit wiederholt auf das Verhältnis seiner Texte untereinander hindeuten. ${ }^{1}$

In seiner dritten Tübinger Poetikvorlesung, „Die Stadt ist kein Völkerkundemuseum“, heißt es: „Alles, was ich je geschrieben habe, ist ein Zeugnis der Zeiten, die ich mitgestaltete und der Zeitlichkeiten, deren Zeuge ich war" (53). Ören versteht sich als eine Art literarischer Chronist und weist auf die Evokation multipler und sich verändernder Zeitlichkeiten in seinem Werk hin, wobei er seine aktive Rolle bei deren Gestaltung unterstreicht. Örens ,,poetisches Denken" ist durch eine dynamische Wechselwirkung verschiedener Zeitlichkeiten gekennzeichnet, die in seiner literarischen Praxis durch die Ansiedlung von Bildern seiner türkischen Vergangenheit in ,,verschiedenen Zeiten“ seiner deutschen Gegenwart sichtbar wird (,Vorstellungskraft“ 8; 12). ,In ganz andere Zusammenhänge gebracht“, schreibt Ören, ,,erzeugen sie neue Spannungen und gewinnen den Texten einen neuen Wahrheitsgehalt ab" (,Vorstellungskraft" 12). Durch diese literarische Methode stellt Ören Verbindungen zwischen türkischen und deutschen Zeitlichkeiten, Geschichten und Kontexten her, um die türkisch-deutsche Gegenwart zu reflektieren. Angesichts seiner Doppelrolle als „Teilnehmer“ und „Zeuge“ im Hinblick auf die türkische Migration nach (West)Deutschland ist die Einwanderung ein wichtiger Impuls und ein zentrales Thema in seinem literarischen Werk. Laut Ören wird durch den Einwanderungsprozess eine andere Zeit „,in die Stadt getragen”, durch die ,neue gesellschaftliche Strukturen“ entstehen (,Selbstbild“ 20). Mit einem sichtbaren Schwerpunkt auf der Einwanderung als Prozess, durch den neue Kontexte und Geschichten eingeführt werden, 
hebt Ören gleichzeitig die Bedeutung der türkischen Vergangenheit(en) für die deutsche Gegenwart und Zukunft hervor. So sollten die drei Teile der Berliner Trilogie laut Ören ,die Neuankömmlinge mit ihren Einzelschicksalen mit den Berlinern zusammenbringen. Ich bezweckte die Empfindungen ihrer Zeitlichkeit zu übersetzen. Es war die Aufgabe eines Übersetzers, die ich übernahm“ („Völkerkundemuseum“ 48). Der Prozess des Übersetzens wird an die Notwendigkeit einer reziproken Annäherung der türkischen und deutschen Seite gekoppelt und durch Örens literarische Texte (wie am Beispiel der Berliner Trilogie verdeutlicht) ermöglicht.

Aras Ören war seit Beginn an der Diskussion zur Verortung seiner literarischen Werke beteiligt, ebenso wie seine Kolleg*innen wie beispielsweise Yüksel Pazarkaya, um nur einen zu nennen. In ihrem richtungsweisenden kritischen Beitrag zur sogenannten Ausländerliteratur hat die Literaturwissenschaftlerin Heidrun Suhr diesen Prozess als „Synchronizität der literarischen Entwicklung und theoretischen Diskussion" bezeichnet (76). ${ }^{2} 1985$ war Aras Ören zusammen mit dem syrisch-deutschen Schriftsteller Rafik Schami der erste Preisträger des Adelbert-von-Chamisso-Preises, der von der RobertBosch-Stiftung ,für bedeutende Beiträge zur deutschen Literatur von Autoren nichtdeutscher Muttersprache" verliehen wurde (Weinrich 11). In seiner Dankesrede charakterisierte Ören das Ziel seines Schreibens als „Ausschau zu halten nach Neuland, dieses zu erschließen und zu bestellen, [...] die Rolle der Literatur neu zu bestimmen. [. . . ] Ein wichtiger Antrieb, davon bin ich fest überzeugt, kann heutzutage von uns Ausländern in der deutschen Literatur kommen, und wir stehen zu dieser Aufgabe“ („Dankrede“ 26). Hier verweist er auf die katalytische Funktion von Schriftsteller*innen nichtdeutscher Herkunft, die in Bezug auf die deutsche Literaturlandschaft nicht nur einen wichtigen Beitrag leisten, sondern diese auch nachhaltig transformieren. Diesbezüglich hebt er wiederholt die Auseinandersetzung mit der deutschen Literatur und ihren literarischen Traditionen hervor und argumentiert gegen eine „Gettoisierung“ der Literatur von Schriftsteller*innen nichtdeutscher Herkunft. Durch eine Klassifikation als Ausländer- oder Gastarbeiterliteratur würde ein literarischer Text nämlich lediglich auf Grund der Herkunft oder des ökonomischen Status des Autors oder der Autorin kategorisiert (,Würde“ 90-93). Ören sieht insbesondere die in Deutschland entstehende türkische Literatur ,als einen integrierten und eigenständigen Bestandteil der deutschen Literatur in der Bundesrepublik und in West-Berlin [...]. [Sie] setzt sich sowohl mit der eigenen türkischen, als auch mit deutschen Traditionen auseinander" (,Völkerkundemuseum“ 52). Auf diese Weise werden mehrere literaturgeschichtliche Zeitlichkeiten - die sowohl die türkische als auch die deutsche Kulturgeschichte betreffen - als Grundlage seiner literarischen Interventionen sichtbar.

Über diese zentrale Rolle hinaus, die Ören Schriftsteller*innen nichtdeutscher Herkunft zuschreibt, wird die türkische Einwanderung nach Deutschland, einschließlich seiner eigenen Migration, an den Schreibprozess 
gekoppelt: „Diese Zeit meiner schriftstellerischen Tätigkeit“, so Ören, „,ist eng verbunden mit den verschiedenen Einwanderungswellen. Beide verliefen parallel zueinander, reflektierten sich. Das Bewusstsein der Einwanderung und mein literarisches Schaffen stehen in ständiger Wechselwirkung, bedingen sich gegenseitig“ (,Dankrede“ 27). Ören nimmt türkische Einwander*innen, die in seinen frühen Texten vorwiegend als handelnde Subjekte auftreten und auch ein wichtiges Thema in seiner Poetik darstellen, ähnlich wie Schriftsteller*innen nichtdeutscher Herkunft, als impulsstiftende Akteur*innen wahr. An dieser Stelle möchte ich Örens Selbstbild als Chronist erneut hervorheben: Indem insbesondere seine frühen Gedichte die Ankunft der türkischen Arbeiter*innen darstellen und weitere Einblicke in den türkischen Kontext der Arbeitsmigration geben, verorten sie die türkischen Arbeitsmigrant*innen im Kontext der deutschen Arbeitsbedingungen und der Proteste der Nachkriegszeit. Ören versteht das Schreiben als „,historische Verantwortung“(,Selbstbild“ 40) und hat in seiner literarischen Arbeit türkischen Arbeiter*innen eine bedeutende Rolle bei der Wiederherstellung einer einheitlichen deutschen Arbeiterbewegung in Westberlin zugewiesen, die nach „Faschismus und Krieg“ ohne Einheit ist (,Glückseligkeit“ 9). Sein frühes literarisches Schaffen, in prosaischer, lyrischer und dramatischer Form, ist daher thematisch nicht nur von einem Fokus auf die Einwanderung türkischer Arbeiter*innen, sondern auch deren Begegnungen mit den westdeutschen Arbeiter*innen geprägt. Ören war aktives Mitglied der literarischen Linken Westberlins und trug sowohl zu ihren ästhetischen Debatten als auch zu ihren institutionellen Kulturen bei. In seinem literarischen Oeuvre schrieb Ören nicht nur über türkische Arbeiter*innen und ihre Erfahrungen, sondern auch über die Solidarität der Arbeiterklasse im Allgemeinen und reflektierte die Ausbeutung der Arbeits- und Produktionsbedingungen in Westberlin. In den frühen 1970er Jahren schloss sich Ören der Roten Nelke, einer antifaschistischen Vereinigung fortschrittlicher Künstler*innen, mit der Zielsetzung an, den Klassenkampf durch Kunst zu unterstützen und zu fördern. Innerhalb der Roten Nelke war Ören Vorsitzender des Arbeitskreises „Gruppe der schreibenden Arbeiter", der sich darum bemühte, sowohl Arbeiter*innen als auch Künstler*innen in ihre kulturpolitischen Aktivitäten und den literarischen Schreibprozess einzubeziehen. Er war aktiv an Diskussionen über die Politisierung von Literatur, das Konzept operativer Kunst, die Politik der Ästhetik und die soziale Verantwortung von Künstler*innen beteiligt. Über den Kontext der Westberliner Arbeiterkultur, linker öffentlich-literarischer Proteste und transnationaler Solidaritätsbewegungen, ist Örens literarisches Schaffen auch in der Politisierung des Theaters der Türkei und Westdeutschlands zu verorten. Sein Engagement innerhalb der westdeutschen Linken, wie auch des Theaters, möchte ich als Zeitlichkeiten - im Sinne Örens, als wesentliche, jedoch kaum berücksichtigte - Kontexte für Örens Werk besonders hervorheben. 
Die 1960er Jahre markieren eine für die Politisierung des türkischen Theaters höchst relevante Zeit, in der Aras Ören „,sowohl in politischen Theorien wie auf den Theaterbühnen nach einer Heimat gesucht" hat (,Vorstellungskraft" 20). Seine Laufbahn im Theater begann 1959 in Istanbul am Gençlik Tiyatrosu, und in den 1960er Jahren arbeitete Ören als Schauspieler und Dramaturg an verschiedenen Theatern in Istanbul, Westberlin und Frankfurt (Main). Das studentische Theaterfestival Erlangen sei hier als ein zentrales Forum für den türkisch-deutschen Kulturaustausch genannt. Eine Reihe türkischer Künstler*innen und Theatermacher*innen, die später nach Westdeutschland ins Exil gingen, wie zum Beispiel Vasıf Öngören, nahmen mehrfach teil. Das 1949 gegründete Studententheaterfestival in Erlangen ermöglichte fast zwei Jahrzehnte lang einen internationalen Dialog über politische Theaterpraktiken zwischen Ensembles, die auf beiden Seiten des Eisernen Vorhanges beheimatet waren. Türkische Ensembles nahmen seit 1954 regelmäßig teil und waren bis zur Auflösung des Festivals 1968 fast jedes Jahr vertreten. Ihre Teilnahme am studentischen Theaterfestival in Erlangen ermöglichte Mitgliedern türkischer Ensembles, Aras Ören eingeschlossen, einen Austausch mit ost- und westdeutschen Theatermacher*innen sowie Mitgliedern der Neuen Bühne Frankfurt und des Berliner Ensemble.

Örens literarischer Durchbruch gelang ihm 1973 mit der Veröffentlichung des ersten Teils seiner Berliner Trilogie oder seines poetischen Zyklus: Was will Niyazi in der Naunynstraße. 1980 wurden alle drei Teile als Trilogie mit dem Titel Berlin Üçlemesi (Berliner Trilogie) in türkischer Sprache veröffentlicht, was darauf hinweist, dass Ören sowohl ein türkischsprachiges als auch ein deutschsprachiges Publikum ansprechen wollte. Die Trilogie wurde in der Reihe „Rotbücher“ veröffentlicht, die vom einzigen kollektiv geführten, linken Verlag in Westberlin, dem Rotbuch Verlag, herausgegeben wurde. Die Neuauflage der Berlin Trilogie, erschienen 2019 im Verbrecher Verlag, widmet Aras Ören "der ersten und zweiten Generation von Menschen aus der Türkei” (5), denn

sie haben einen unvergesslichen Anteil an unserem heutigen Wohlstand und kulturellen Reichtum [... ] Wir sollten diesen bescheidenen Menschen dankbar dafür sein, daß sie frischen Wind in unsere alte europäische Kulturlandschaft gebracht haben. Sie änderten sich selbst und Europa [...] Ihnen [ihren Kindern] mag die Trilogie dabei helfen, der Geschichte ihrer Väter und Mütter näher zu kommen und sie vor dem Vergessen zu bewahren. Denn auch in der Gegenwart schwingt die Vergangenheit stets mit. (6)

Die Berlin Trilogie wird als eine Art Chronik umschrieben, ein literarischhistorisches Zeitdokument, das ermöglichen soll, das Vergangene im Gegenwärtigen zu erkennen und dabei dem Vergessen vergangener Zeitlichkeiten - insbesondere im Kontext der türkischen Migration - entgegen zu wirken.

Das literarische Erfassen und Zusammenführen verschiedener Zeitlichkeiten, einschließlich die der türkischen Migration, geht über die literarische 
Tätigkeit Örens hinaus. Als Schauspieler, Dramatiker, Schriftsteller, Dichter, Theoretiker und Radiojournalist nahm er regelmäßig an zahlreichen kulturellen Veranstaltungen teil. Auch als Mitgründer und Redakteur des ersten regulären türkischsprachigen Radioprogramms in Westdeutschland, das vom SFB (Sender Freies Berlin) ausgestrahlt wurde und sich auf das türkische Leben in Westberlin konzentrierte, war er eine wichtige Persönlichkeit des öffentlichen Lebens. Darüber hinaus war Ören in mehreren kulturellen Gremien aktiv (wie beispielweise in der Senatsverwaltung für kulturelle Angelegenheiten und im Beirat für Kulturaustausch) und hat die türkische Kultur in Westberlin nicht nur nachhaltig geprägt, sondern diese Aktivitäten detailliert dokumentiert. Dank seines Vorlasses in der Akademie der Künste Berlin, auf den Helga Neumanns Beitrag (,Archive sind unfreundlich von soviel aufbewahrter Wirklichkeit." Zum Aras-Ören-Archiv in der Akademie der Künste, Berlin) näher eingeht, ist es möglich, türkische Kulturpraktiken und - aktivitäten seit den frühen 1970er Jahren zu rekonstruieren.

Dieses Sonderheft soll das literarische Schaffen Aras Örens sowie seine kulturpolitischen Beiträge in den Vordergrund rücken und gleichzeitig ihre anhaltende Bedeutung hervorheben. Es bringt renommierte Wissenschaftler*innen aus verschiedenen institutionellen und nationalen Kontexten zusammen und bietet nicht nur neue Ansätze für Örens Arbeit, sondern umfasst auch ausgewählte erstmalige Übersetzungen ins Englische (von Yasemin Yildiz und Tom Cheesman), die seine Leser*innenschaft erweitern und somit die Möglichkeit bieten, Örens Texte in englischsprachige Kurse zu Themen wie Migration, Rassismus, Staatsbürgerschaft, Europa, Arbeiterklasse und Berlin aufzunehmen.

Darüber hinaus ermöglicht der Aufsatz von Helga Neumann einen Einblick in Aras Örens Vorlass. Örens sorgfältige Sammlung und chronologische Archivierung von Zeitungsartikeln in türkischer und deutscher Sprache, von Programmbroschüren, Korrespondenzen (zwischen Kulturträger*innen, Künstler*innen und Institutionen), Theatermanuskripten und Veranstaltungsankündigungen ermöglicht die Rekonstruktion eines vielfältigen türkischen Kulturprogramms vor 1989. Gleichzeitig gewähren die von Ören gewissenhaft zusammengetragenen Materialien auch Einblick in die Konzeptualisierung und Finanzierung der türkischen Kultur (von Seiten der Künstler*innen, Kulturinstitutionen und dem Westberliner Senat) in den frühen Jahren der türkischen Einwanderung. Diese erfolgte nicht nur im Rahmen des Rotationsprinzips und des Anwerbeabkommens, sondern auch durch politisch verfolgte Intellektuelle und Künstler*innen.

Leslie Adelsons Beitrag ,When Sincerity Fails: Literatures of Migration and the Emblematic Labor of Personhood" - ein Nachdruck aus ihrem bahnbrechenden Buch The Turkish Turn in Contemporary German Literature (2005) - untersucht, wie Literaturen der türkischen Migration, hier Örens Novelle Bitte nix Polizei (1981), Tropen der ethnischen - und Genderzuge- 
hörigkeit, die das kulturelle Kapital der Migration prägen, neu konfigurieren und dabei die Zusammensetzung ethnischer Räume neu denken. In „Intermedial Solidarity: Drawing Inspiration from the 1970s“ geht Deniz Göktürk intermedialen und intertextuellen Bezügen in dem Dokumentarfilm Frau Kutzer und andere Bewohner der Naunynstraße (1973) - eine Kollaboration zwischen Ören und dem Regisseur Friedrich W. Zimmermann - nach, mit einem Schwerpunkt auf Örens audiovisueller Poetik und multimedialer Ästhetik. David Gramlings Beitrag, ,Back by Inscrutable Demand: Ali Itır's Multilingual Return in Berlin Savignyplatz", nimmt eine intertextuelle Lektüre von Örens Roman Berlin Savignyplatz (1995) vor, in dem sowohl die türkische als auch die deutsche Version (letztere übersetzt von Deniz Göktürk) auf ihre jeweilige Resonanz der Figur Ali Itrr aus Örens früherem Roman Bitte nix Polizei im Rahmen seiner multilingualen Poetik untersucht wird. Moray McGowan greift in "The Hand that Rocks the Cradle? Aras Ören's ,Europa“" Örens frühere Texte erneut auf, um aus der gegenwärtigen Perspektive die anhaltende Relevanz der sozialen Erfahrung von Arbeitnehmer*innen und anderen Migrant*innen innerhalb der gesamteuropäischen Erfahrungen der Arbeiterklasse in Bezug auf Örens literarische Konstruktion Europas zu untersuchen. Yasemin Yildiz und Tom Cheesman tragen erstmals ins Englische übersetzte Texte Örens bei, die durch kritische Anmerkungen eingeleitet werden und zugleich die andauernde Relevanz dieser frühen richtungsweisenden Texte reflektieren. Yasemin Yildiz übersetzt in „Aras Ören's What Does Niyazi Want in Naunyn Street: A Partial Translation with a Translator's Introduction“ Auszüge des ersten Teils der Berlin Trilogie. In „What's Going On: Poems by Aras Ören with a Translator's Introduction" überträgt Tom Cheesman frühere Gedichte aus der 2017 erschienenen Anthologie Wir neuen Europäer. Ein Lesebuch ins Englische. ${ }^{3}$

Als Gast-Herausgeberin möchte ich mich bei allen Kolleg*innen für ihre enthusiastische Unterstützung und Mitarbeit an diesem Sonderheft und ihre anregenden Beiträge bedanken. Besonderer Dank gebührt auch den Monatshefte Herausgeberinnen, Hannah Vandegrift Eldridge und Sonja Klocke, für ihr Interesse und Engagement und vor allem für die Bereitstellung eines Forums für die Veröffentlichung der folgenden Beiträge. Dieser Sonderband ist Aras Ören gewidmet, dessen ,Zeugnis[sse] der Zeiten, die [er] mitgestaltete und der Zeitlichkeiten, deren Zeuge [er] war“ (,Völkerkundemuseum" 53) uns noch lange begleiten und inspirieren werden.

${ }^{1}$ Dieser Beitrag beruht auf meiner früheren literaturwissenschaftlichen Arbeit zu Aras Ören, siehe Gezen (2016) und Gezen (2018).

${ }^{2}$, ,[...] synchronicity of literary development and theoretical discussion." Meine Übersetzung.

${ }^{3}$ Für die Freigabe der Rechte zur Übersetzung gilt unser Dank den Kolleg*innen im Verbrecher Verlag, insbesondere Kristine Listau und Jörg Sundermeier. 


\title{
Zitierte Literatur
}

Gezen, Ela. “Aras Ören and the (West)German Literary Left.” Literature Compass 13.5 (2016): 324-331. Print.

Gezen, Ela. Brecht, Turkish Theater, and Turkish-German Literature: Reception, Adaptation, and Innovation after 1960. Rochester: Camden House, 2018. Print.

Ören, Aras. ,Die Glückseligkeit eines Herrn Otto Schulze.' Asphalt 6 (1971): 9. Print.

Was will Niyazi in der Naunynstraße. Ein Poem. Aus dem Türkischen von H. Achmed Schmiede und Johannes Schenk. Berlin: Rotbuch Verlag, 1973. Print. Print. Privatexil. Gedichte. Aus dem Türkischen von Gisela Kraft. Berlin: Rotbuch, 1977.

Berlin Üçlemesi: Poem. Istanbul: Remzi Kitabevi, 1980. Print.

„,Von der Würde des Künstlers gegenüber dem missionarisch-bürokratischen Egoismus.“ Eine nicht nur deutsche Literatur. Zur Standortbestimmung der „Ausländerliteratur. “ Hg. Irmgard Ackermann und Harald Weinrich. München: Piper, 1986. 90-93. Print.

—. „Dankrede zur Preisverleihung.“ Chamissos Enkel. Literatur von Ausländern in Deutschland. Hg. Heinz Friedrich. München: Deutscher Taschenbuch Verlag, 1986. 25-29. Print.

Dazwischen. Gedichte. Aus dem Türkischen von Helga Dağyeli-Bohne und Yıldırım Dağyeli. Frankfurt Main: Dağyeli Verlag, 1987. Print.

. „Vorstellungskraft und Zeit.“ Privatexil: Ein Programm? Drei Vorlesungen. Aus dem Türkischen von Cem Dalaman. Tübingen: Konkursbuchwelt, 1999. 5-21. Print.

_. „Selbstbild mit Stadt.“ Privatexil: Ein Programm? Drei Vorlesungen. Aus dem Türkischen von Cem Dalaman. Tübingen: Konkursbuchwelt, 1999. 23-41. Print.

— ., Eine Metropole ist kein Völkerkundemuseum.“Privatexil: Ein Programm? Drei Vorlesungen. Aus dem Türkischen von Cem Dalaman. Tübingen: Konkursbuchwelt, 1999. 4360. Print.

Suhr, Heidrun. „Ausländerliteratur: Minority Literature in the Federal Republic of Germany.“ New German Critique 46 (Winter, 1989): 71-103. Print.

Weinrich, Harald. „Der Adelbert-von-Chamisso-Preis.“ Chamissos Enkel. Literatur von Ausländern in Deutschland. Hg. Heinz Friedrich. München: Deutscher Taschenbuch Verlag, 1986. 11-13. Print.

\author{
Ela Gezen \\ University of Massachusetts Amherst \\ 161 Presidents Drive \\ Amherst, MA01003 \\ egezen@german.umass.edu
}

